\title{
The Role of Medical Therapy in Idiopathic Ventricular Fibrillation
}

\author{
Chiara Scrocco, Bode Ensam, Elijah R Behr \\ Cardiology Clinical Academic Group, St. George's, University of London and St. George's University Hospitals NHS Foundation Trust, London, UK
}

diopathic ventricular fibrillation (VF) is a diagnosis of exclusion following a resuscitated cardiac arrest which remain unexplained after comprehensive cardiac, respiratory, metabolic and toxicological evaluation. However, idiopathic VF patients may share electrophysiological

and clinical features. Polymorphic ventricular tachycardia, rapidly degenerating in $\mathrm{VF}_{\mathrm{F}}$ is often triggered by short-coupled premature ventricular contractions originating from the Purkinje system or localised areas of the inferior left or right ventricular wall. Secondary prevention with an automated implantable cardiac defibrillator is a cornerstone in idiopathic VF treatment. Ablation strategies may also play a role where a substrate is identified. The role of medical therapy in idiopathic VF is, however, poorly defined, mainly due to the lack of exploratory studies and the absence of randomised clinical trials. Use of IV calcium channel antagonists and isoprenaline for the acute management of VF has proven effective in idiopathic VF associated with short-coupled Torsades de Pointes and early repolarisation, respectively. Despite the lack of large-scale data, long-term prophylaxis with quinidine seems to be effective in suppressing VF recurrence, regardless of the associated electrocardiogram phenotype. Further understanding of the underlying mechanisms of idiopathic VF, including genetic studies, will play a role in the development of targeted pharmacological therapies.

\section{Keywords}

Idiopathic ventricular fibrillation, sudden death, cardiac arrest, ventricular tachycardia, quinidine, isoproterenol

Disclosure: Chiara Scrocco, Bode Ensam and Elijah R Behr have nothing to declare in relation to this article.

Review Process: Double-blind peer review.

Compliance with Ethics: This study involves a review of the literature and did not involve any studies with human or animal subjects performed by any of the authors.

Authorship: The named authors meet the International Committee of Medical Journal Editors (ICMJE) criteria for authorship of this manuscript, take responsibility for the integrity of the work as a whole, and have given final approval for the version to be published.

Received: 16 August 2019

Accepted: 18 October 2019

Citation: European Journal of Arrhythmia

\& Electrophysiology. 2019;5(2):87-91

Corresponding Author: Elijah R Behr,

Cardiology Clinical Academic Group, St George's

University Hospitals' NHS Foundation Trust

and Molecular and Clinical Sciences Institute,

St George's University of London, Cranmer Terrace,

London SW17 ORE, UK. E: ebehr@sgul.ac.uk

Support: No funding was received in

the publication of this article.
A survivor of cardiac arrest due to ventricular fibrillation (VF), without a cardiac, respiratory, metabolic, or toxicological explanation, is diagnosed with idiopathic VF.' Idiopathic VF is estimated to account for approximately 5-7\% of all out-of-hospital cardiac arrest cases. ${ }^{2,3}$ Furthermore, the Cardiac Arrest Survivors With Preserved Ejection Fraction Registry (CASPER) showed that $44 \%$ of out-of-hospital cardiac arrests without a clear presenting cause remained unexplained despite extensive investigations of the patient and their family members. ${ }^{4}$ CASPER also emphasised that the re-evaluation of patients with idiopathic VF, over time, may lead to a diagnosis in up to $20 \%$ of cases. ${ }^{5}$ More recent European data on 717 cardiac arrest survivors highlighted that a complete workup, including pharmacological provocation tests, identified diagnoses in most of the cases, with $6.8 \%$ labelled as idiopathic VF. ${ }^{6}$ These findings reflect the importance of comprehensive cardiac evaluation of cardiac arrest victims and family members for accurate diagnosis.

\section{Idiopathic ventricular fibrillation}

The term "idiopathic VF" is a diagnosis of exclusion; however, patients with idiopathic VF often share typical electrophysiological and clinical features. The first use of the term dates back to 1987, with the report by Belhassen of five cardiac arrest survivors (three men and two women, aged from 24-52 years) in whom myocardial ischaemia, metabolic or electrolyte disturbances, drug toxicity, pre-excitation, or prolonged QT interval had been excluded. In all subjects, a rapid poorly tolerated polymorphic ventricular tachycardia (VT) was induced by programmed ventricular stimulation, and in one case, a spontaneous polymorphic VT rapidly degenerating to VF was documented. ${ }^{?}$

In 1990, Leenhardt et al. described the mode of onset of spontaneous arrhythmias in 14 subjects with no structural heart disease and unexplained syncope, which consisted of a single premature ventricular contraction (PVC) with extremely short coupling interval (R-on-T phenomenon). The PVC occurred during a vulnerable period and therefore triggered a rapid polymorphic VT or VF. The electrocardiogram (ECG) pattern was termed "short-coupled Torsades de Pointe" (TdP) to highlight variable electrical activation of the arrhythmia with progressive changes in QRS morphology, amplitude, and polarity. ${ }^{8}$ These findings were confirmed by Viskin et al. in a cohort of unexplained cardiac arrest survivors; in 22 VF episodes recorded in nine subjects, the PVC initiating a rapid polymorphic VT had a coupling interval of $300 \pm 52 \mathrm{~ms}$. The episodes were not preceded by long-short RR sequences. ${ }^{9}$

The highly malignant nature of this non-pause dependent idiopathic polymorphic VT (i.e., not influenced by the sympathetic tone or by coronary hyperreactivity) was described further by Eisenberg et al. in a cohort of 15 idiopathic VF patients. ${ }^{10}$ The study showed that shorter PVC coupling intervals correlated with greater risk of spontaneous polymorphic VT and sudden cardiac death. The advent of electro-anatomical mapping techniques has allowed accurate identification 
Figure 1: Short-coupled premature ventricular contractions with R-on-T phenomenon triggering a non-sustained polymorphic ventricular tachycardia in a 32-year-old female idiopathic ventricular fibrillation survivor

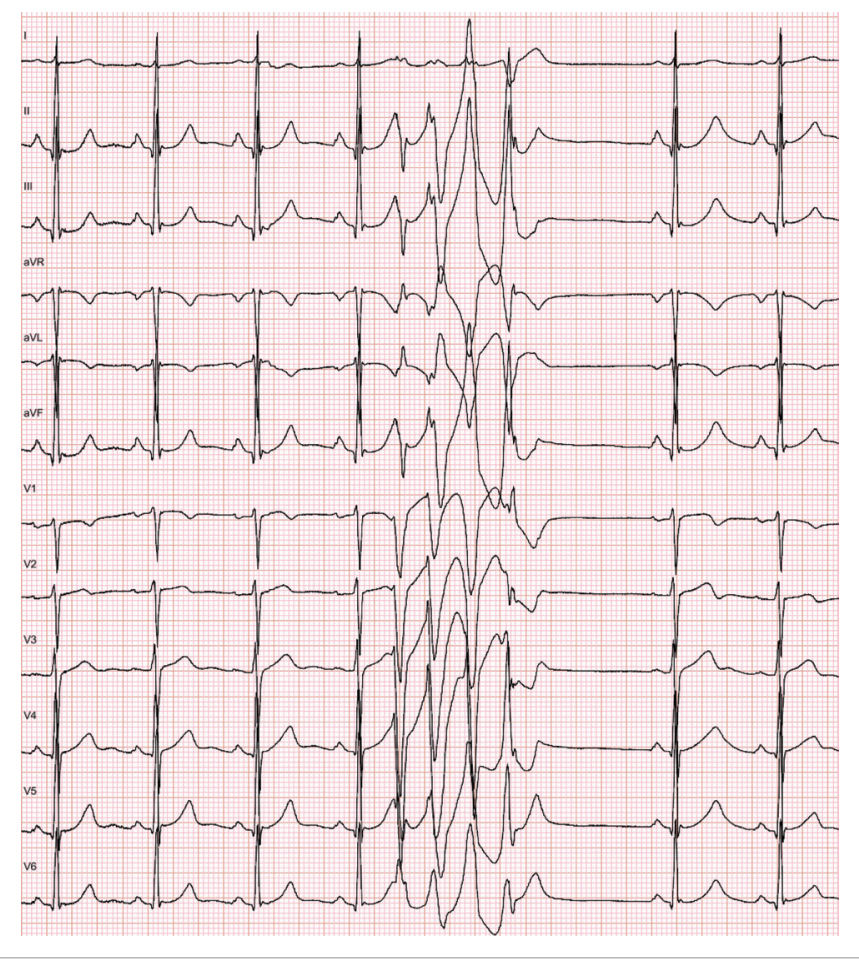

of the site of origin of PVCS triggering VF in unexplained cardiac arrest survivors. Haïssaguerre and colleagues found that the foci are localised in the specialised Purkinje system in more than $85 \%$ of cases. These Purkinje beats, similar in morphology to fascicular tachycardias from the left ventricle or right ventricle, usually exhibited a short coupling interval. ${ }^{11,12}$ An example of short-coupled PVC triggering non-sustained polymorphic VT is shown in Figure 1.

In the last decade, case-control and epidemiological studies have described the association between J waves, defined as positive deflections immediately following the QRS complex and idiopathic VF. ${ }^{13-15}$ The presence of a J wave in the inferolateral ECG leads, which may or may be not be followed by ST segment elevation, is known as early repolarisation pattern. Early repolarisation pattern is a common ECG finding (estimated incidence 1-13\%), usually considered innocent amongst healthy asymptomatic young individuals. ${ }^{1}$

Haïssaguerre et al. found that early repolarisation pattern was present in $31 \%$ of 206 case subjects with idiopathic VF cases and $5 \%$ of 412 matched subjects without heart disease; moreover, idiopathic VF subjects with early repolarisation pattern had a higher incidence of recurrent VF at follow-up (hazard ratio 2.1). ${ }^{13}$ The link between early repolarisation pattern and malignant arrhythmias is supported by the accentuation of the J wave before the onset of $\mathrm{VF}$, and the observation of triggering PVCs coincident with the J wave at ECG. J waves are also associated with VF storms, defined as $\geq 3$ VF episodes in 24 hours. . $^{13,16,17}$ The term early repolarisation syndrome has increasingly been used to identify patients with idiopathic VF and early repolarisation pattern at ECG (Figure 2A).

The pathophysiology underlying early repolarisation syndrome is, however, still debated. According to animal models supported by
Figure 2: Idiopathic ventricular fibrillation associated with inferior J waves (early repolarisation syndrome)
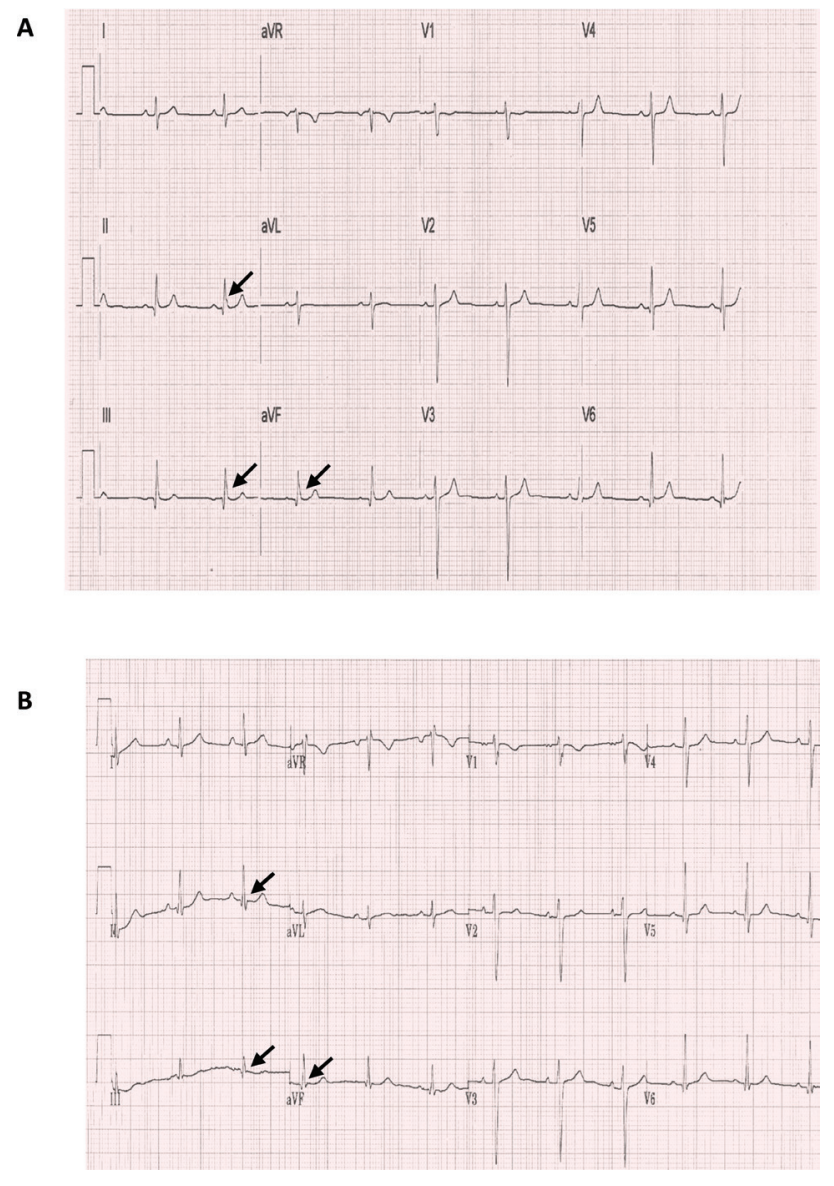

A. Idiopathic ventricular fibrillation associated with inferior J wave (arrows) in a 39-year-old male; $B$. suppression of the inferior J wave (arrows) after oral hydroquinidine $300 \mathrm{mg}$ twice daily.

ECG imaging studies, enhanced inward ion currents during phase 2 of the action potential (AP) are responsible for premature myocardial repolarisation. ${ }^{18,19}$ Increasing evidence supports an alternative hypothesis, according to which the J point elevation typical of early repolarisation pattern could be an expression of delayed depolarisation. High-density mapping studies in some early repolarisation syndrome survivors showed delayed, fragmented epi- and endocardial ECGs indicating local structural alterations in the inferior right and/or left ventricular walls coincident with the J wave. ${ }^{20}$ Similar findings have also been reported in a study involving 24 patients with no electrocardiographic phenotype. Localised areas of abnormal depolarisation were identified in $62 \%$, highlighting the increasing role of depolarisation defects, with or without ECG manifestation, in the pathophysiology of sudden cardiac death in apparently normal hearts. ${ }^{21}$

\section{Medical management of idiopathic ventricular fibrillation}

Both guidelines and expert consensus documents agree that implantable cardiac defibrillator (ICD) implantation is recommended in patients with a diagnosis of idiopathic VF (class I). Optimal pharmacological treatment is less well defined due to the low prevalence of the condition and the absence of randomised case control studies. ${ }^{1,22}$ Table 1 summarises the current recommendations for medical therapy in idiopathic $\mathrm{VF}^{1,22}$ The first drug trial in idiopathic VF was described by Belhassen and colleagues almost 30 years ago; in two of the first five described patients 
Table 1: Current recommendations for the medical treatment of idiopathic ventricular tachycardia

\begin{tabular}{|l|l|}
\hline $\begin{array}{l}\text { Current recommendations for the medical treatment of } \\
\text { idiopathic ventricular tachycardia }\end{array}$ & $\begin{array}{l}\text { Level of } \\
\text { recommendation }\end{array}$ \\
\hline $\begin{array}{l}\text { Intravenous verapamil to acutely suppress/prevent an } \\
\text { electrical storm or recurrent ICD discharges should be } \\
\text { considered in short-coupled TdP }\end{array}$ & $\mathrm{Il}^{22}$ \\
\hline $\begin{array}{l}\text { Isoproterenol infusion can be useful in suppressing electrical } \\
\text { storms in patients with a diagnosis of ERS }\end{array}$ & $\| \mathrm{I}^{1}$ \\
\hline $\begin{array}{l}\text { Quinidine in addition to an ICD can be useful for secondary } \\
\text { prevention of VF in patients with a diagnosis of ERS }\end{array}$ & $\mathrm{Il}^{1}$ \\
\hline $\begin{array}{l}\text { Antiarrhythmic therapy with quinidine, PES-guided or } \\
\text { empirical, may be considered in patients with a diagnosis of } \\
\text { idiopathic VF in conjunction with ICD implantation or when } \\
\text { ICD implantation is contraindicated or refused }\end{array}$ & \\
\hline
\end{tabular}

$E R S$ = early repolarisation syndrome; $I C D$ = implantable cardioverter defibrillator; $P E S=$ programmed electrical stimulation; $T d P=$ Torsades de Pointes;

$V F=$ ventricular fibrillation

with inducible VF at electrophysiology study, electrical stimulation was repeated after intravenous disopyramide administration. In one case, disopyramide was effective in suppressing arrhythmias inducibility and long-term prophylaxis started (600 mg/daily), while in the other subject a self-terminating VT/VF could still be induced and disopyramide was substituted with quinidine. Four patients began long-term prophylaxis with oral quinidine (mean dosage 1,650 mg/daily) alone or, in two cases, combined with amiodarone. All patients remained asymptomatic during a mean follow-up of 52 months.? The long-term efficacy of quinidine was confirmed by the same group over 20 years later in nine subjects with idiopathic VF or Brugada syndrome with previous cardiac arrest or syncope. Over a mean follow-up of $15 \pm 7$ years no recurrences of arrhythmias were documented and repeat electrophysiological studies failed to reproduce any sustained arrhythmias. ${ }^{23}$

\section{Short-coupled Torsades de Pointe/polymorphic ventricular tachycardia}

Only two case series studies, to date, described the response to antiarrhythmic agents in idiopathic VF cases triggered by short-coupled PVCS. In the study by Leenhardt et al. ${ }^{8}$ isoproterenol and atropine had mixed effects on suppressing short-coupled PVCs. During follow-up, two subjects treated with betablockers and one treated with verapamil died of sudden cardiac death. Overall, verapamil suppressed arrhythmia recurrence in seven out of 12 subjects. Verapamil, a slow calcium channel blocking agent, acts mostly on the sinoatrial (SA) and atrioventricular (AV) nodes causing depression of automaticity, slowing of conduction and increase in refractoriness. In addition, calcium-channel blockers can ameliorate arrhythmias caused by afterdepolarisations, or by localised areas of slow conduction. The proposed mechanisms for the effectiveness of verapamil in short-coupled TdP were lengthening of the coupling interval and suppression of repetitive PCVs. In the cohort described by Eisenberg et al. ${ }^{10}$ for patients with spontaneous, short-coupled TdP, treatment included both beta-blockers and/or calcium channel blockers; although again, these were not effective in the long term. According to the most recent guidelines, the use of IV calcium channel blockers should be considered for the acute management of VF storms or recurrent ICD discharges in subjects with short-coupled TdP (class IIb). ${ }^{22}$ Importantly, cases of idiopathic VF that responded well to quinidine also seem to be triggered by short-coupled PVCs. ${ }^{723}$ Thus, whilst not formally mentioned in the guidelines, quinidine can also be useful in suppression of PVCs.

\section{Idiopathic ventricular fibrillation associated with J waves (early repolarisation syndrome)}

Haïssaguerre et al. first described the effectiveness of different drug therapies in early repolarisation syndrome in $2009 .{ }^{24}$ In 16 subjects with VF storms, no response was achieved with beta-blockers (tested in 11 subjects), lidocaine/mexiletine (tested in nine subjects), and verapamil (tested in three subjects), while amiodarone was effective in one-third of cases. Isoproterenol infusion (1-5 $\mu \mathrm{g} / \mathrm{min})$ immediately suppressed all arrhythmias in seven patients when the sinus heart rate was increased above 120 beats/minute..$^{24}$

Isoproterenol is a beta-1 and beta-2 adrenergic receptor agonist. The effects on beta- 1 adrenergic receptors, primarily concentrated in the heart, include an increase in intracellular calcium, resulting in a steeper slope of the cardiac pacemaker AP phase 4; therefore, pacemaker cells reach the threshold at a faster rate, resulting in the characteristic decrease in basic cycle length. In the epicardium, this markedly diminishes the spike-and-dome appearance of the AP plateau and causes marked shortening of AP duration, due to a rate-dependent reduction of the transient outward current $\left(\mathrm{I}_{\mathrm{TO}}\right)$ secondary to incomplete recovery from inactivation.

In the endocardium $\mathrm{I}_{\text {TO }}$ currents are weaker, resulting in little or no change in AP duration in response to increases in heart rate. Experimental models of early repolarisation syndrome suggest that cells in the inferior region of ventricular epicardium possess a higher level of $\mathrm{I}_{\text {To }}$ than those in the lateral LV and that this predisposes the inferior region to develop phase 2 re-entry and VT/VF. It also suggests that isoproterenol acts by restoring the epicardial AP dome by causing an inward shift in the balance of current. ${ }^{18}$

In the same case series, the efficacy of different pharmacological treatments over a follow-up of $69 \pm 58$ months was reported. ${ }^{24}$ Prophylaxis with standard antiarrhythmic drugs was poor in preventing recurrent VF: beta-blockers were effective in 2/16 subjects, verapamil in $0 / 4$, mexiletine in $0 / 4$, amiodarone in $1 / 7$, and class IC drugs in 2/9. Only quinidine/hydroquinidine was successful in all tested patients (9/9), decreasing recurrent VF from $33 \pm 35$ episodes to nil. Similar results were obtained in another case study of 10 patients with idiopathic VF and VF storms: isoproterenol was effective in suppressing the arrhythmias and reducing the J waves amplitude at ECG (from $0.493 \pm 0.198 \mathrm{mV}$ prior to VF recurrence to $0.091 \pm 0.101 \mathrm{mV}$ $[p<0.0001])$, while trials with procainamide, lidocaine, verapamil, amiodarone, nifekalant, dofetilide, beta-blockers, and magnesium sulphate were unsuccessful. ${ }^{16}$ Quinidine was also effective, but was tested in only three subjects. Thus, quinidine may be useful, in addition to an ICD, for secondary prevention of VF in patients with a diagnosis of early repolarisation syndrome (class $\| \mathrm{lb}){ }^{1}$

\section{Mechanism of quinidine}

The anti-arrhythmic effects of quinidine are thought, in part, to be due to inhibition of $\mathrm{I}_{\mathrm{TO}}$ and $\mathrm{I}_{\mathrm{Kr}}$ currents causing a prolongation of both AP duration and refractory periods. Quinidine also blocks the fast sodium channel, therefore slowing the phase 0 of the AP and depressing spontaneous phase 4 diastolic depolarisations. Its effects are seen in both atrial and ventricular tissue, and the added anti-vagal action causes acceleration of AV nodal conduction. Quinidine appears effective across idiopathic VF with no definite ECG phenotype, short-coupled TdP, or associated with I waves. Its efficacy in short-coupled TdP could arguably be attributed to its negative dromotropic effect (reduction of conduction velocity) and the 
Figure 3: Effects of quinidine on cardiac action potential

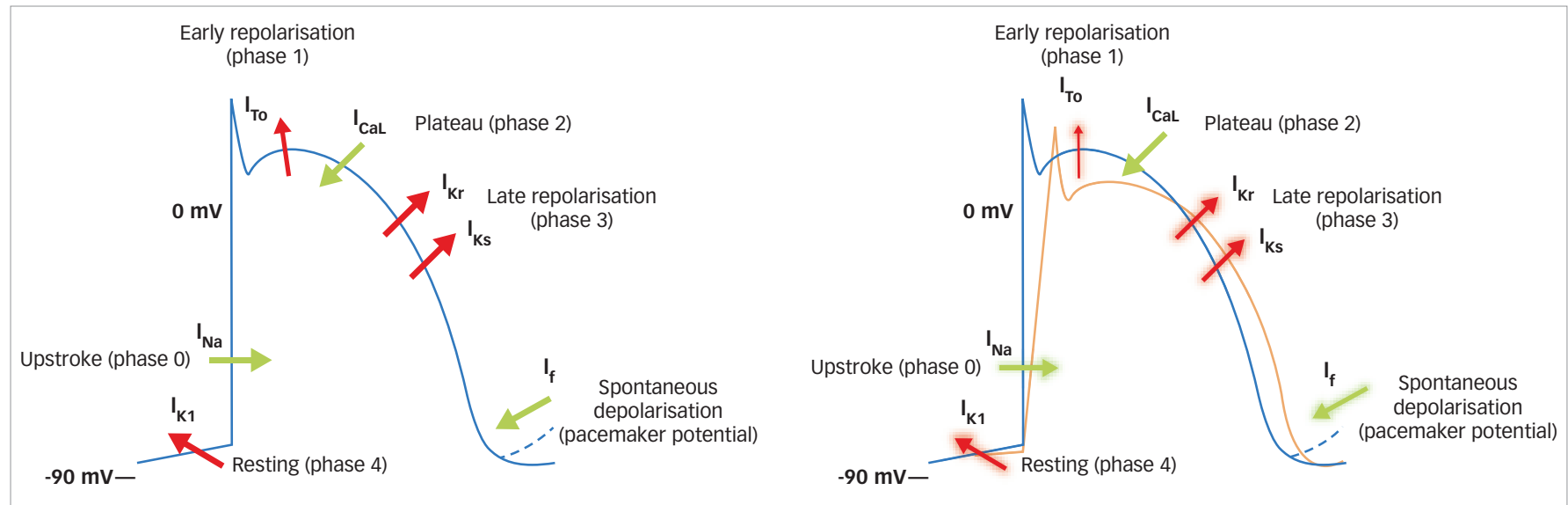

Normal cardiac muscle action potential (blue line) and effects of quinidine (orange line) on the sodium $\left(I_{N A^{\prime}} I_{f}\right)$ and potassium $\left(I_{T O}, I_{K r^{\prime}} I_{K r^{\prime}} I_{K S^{\prime}} I_{f}\right)$ currents. Red arrows show outward currents; green arrows show inward currents. See text for explanation.

prolongation of the refractory period, making myocytes unexcitable by short-coupled PVCs and suppressing automaticity in Purkinje fibres.

There are different theories on the impact of quinidine on the mechanisms of arrhythmogenesis in early repolarisation syndrome. According to the repolarisation theory, a transmural heterogeneity of the AP phase 2 duration, mediated by $I_{\text {TO }}$ currents, leads to a net increase of repolarisation current in the epicardium relative to endocardium, resulting in local re-entry and polymorphic ventricular arrhythmias, providing both the trigger and substrate of $\mathrm{VF}_{.}{ }^{25}$ The $\mathrm{I}_{\mathrm{TO}}$ blocking effect of quinidine would restore the phase 2 dome and suppress this vulnerability. The depolarisation theory suggests that slow conduction areas, secondary to fibrosis and discontinuities in conduction, plays a primary role in the development of the ECG and arrhythmic manifestations. Changes in ionic currents (loss of function in $I_{\mathrm{Na}}$ and I-type $I_{\mathrm{Ca}}$ and gain of function of $\mathrm{I}_{\mathrm{TO}}$; Figure 3) reduce the safety of conduction at high-resistance junctions, such as regions of extensive fibrosis or Purkinje fibre ventricular myocyte junctions, by altering the AP morphology during phase 2 as described above. Experimental studies in rabbits showed that $\mathrm{I}_{\text {TO }}$ inhibition significantly enhances conduction between ventricular myocytes, suggesting that $\mathrm{I}_{\text {To }}$ current plays a major functional role in rate-dependent conduction abnormalities. ${ }^{26}$ Moreover, coupling-induced spontaneous activity in the Purkinje can trigger acute arrhythmias by increased gap junctional resistance at the Purkinje-ventricular interface. ${ }^{27}$ In this scenario, the block of $\mathrm{I}_{\mathrm{T}}$ currents and the consequent restoration of a normal AP phase 2 dome would be a key factor for the suppression of arrhythmias by quinidine and the reduction of the J wave amplitude at ECG (Figure 2B).

Interestingly, both isoproterenol and quinidine are also effective in the acute management and long-term prophylaxis of arrhythmias in the Brugada and short QT syndromes. ${ }^{28-31}$ Further insights into the mechanisms of these genetically mediated ion channel diseases could help shed some light on the mechanism underlying early repolarisation syndrome.

\section{Summary}

The pharmacological treatment of idiopathic VF is poorly defined, mainly due to the lack of exploratory drug studies and the absence of randomised clinical trials and large-scale data. However, relatively robust evidence justifies the use of calcium channel antagonists and isoprenaline for the acute management of VF triggered by short-coupled TdP and associated with early repolarisation, respectively. Long-term prophylaxis with quinidine appears to be safe and effective in suppressing VF recurrence, regardless of the associated ECG phenotype. Further understandings on the underlying mechanisms of idiopathic VF, including genetic studies, will undoubtedly play a role in the development of targeted pharmacological therapies. $\square$
1. Priori SG, Wilde AA, Horie M, et al. HRS/EHRA/APHRS expert consensus statement on the diagnosis and management of patients with inherited primary arrhythmia syndromes: document endorsed by HRS, EHRA, and APHRS in May 2013 and by ACCF, AHA, PACES, and AEPC in June 2013 Heart Rhythm. 2013;10:1932-63.

2. Zipes D, Wellens HJ. Sudden cardiac death. Circulation. 1998;98:2334-51.

3. Survivors of out-of-hospital cardiac arrest with apparently normal heart. Need for definition and standardized clinica evaluation. Consensus Statement of the Joint Steering Committees of the Unexplained Cardiac Arrest Registry of Europe and of the Idiopapathic Ventricular Fibrillation Registry of the United States. Circulation. 1997;95:265-72.

4. Krahn AD, Healey JS, Chauhan V, et al. Systematic assessment of patients with unexplained cardiac arrest: Cardiac Arrest of patients with unexplained cardiac arrest: Cardiac Arrest
Survivors with Preserved Ejection Fraction Registry (CASPER). Survivors with Preserved Ejection
Circulation. 2009:120:278-85.

5. Matassini MV, Krahn AD, Gardner M, et al. Evolution of clinical diagnosis in patients presenting with unexplained cardiac arrest or syncope due to polymorphic ventricular tachycardia. Heart Rhythm. 2014;11:274-81.

6. Waldmann V, Bougouin W, Karam N, et al. Characteristics and clinical assessment of unexplained cardiac arrest in the real-world setting: focusing on idiopathic ventricular fibrillation Eur Heart J. 2018;39:1981-7.

7. Belhassen B, Shapira I, Shoshani D, et al. Idiopathic ventricular fibrillation: inducibility and beneficial effects of class I antiarrhythmic agents. Circulation. 1987:75:809-16.

8. Leenhardt A, Glaser E, Burguera M, et al. Short-coupled variant of torsade de pointes. A new electrocardiographic entity in the spectrum of idiopathic ventricular tachyarrhythmias Circulation. 1994;89:206-15.

9. Viskin $\mathrm{S}$, Lesh MD, Eldar M, et al. Mode of onset of malignant ventricular arrhythmias in idiopathic ventricular fibrillation. J Cardiovasc Electrophysiol. 1997;8:1115-20.

10. Eisenberg SJ, Scheinman MM, Dullet NK, et al. Sudden cardiac death and polymorphous ventricular tachycardia in patients with normal QT intervals and normal systolic cardiac function. Am I Cardiol. 1995;75:687-92.

11. Haïssaguerre $M$, Shah $D C$, Jaïs $P$, et al. Role of Purkinje conducting system in triggering of idiopathic ventricular fibrillation. Lancet. 2002;359:677-8

12. Haïssaguerre M, Shoda M, Jaïs P, et al. Mapping and ablation of idiopathic ventricular fibrillation. Circulation. 2002;106:962-7.

13. Haïssaguerre M, Derval N, Sacher F, et al. Sudden cardiac arrest associated with early repolarization. N Eng/ J Med. 2008:358:2016-23.

14. Rosso R, Kogan E, Belhassen B, et al. J-point elevation in survivors of primary ventricular fibrillation and matched control subjects. Incidence and Clinical Significance. J Am Coll Cardiol. 2008;52:1231-8.

15. Tikkanen JT, Anttonen O, Junttila MJ, et al. Long-term outcome associated with early repolarization on electrocardiography. N Engl J Med. 2009;361:2529-37.

16. Aizawa $Y$, Chinushi M, Hasegawa K, et al. Electrical storm in idiopathic ventricular fibrillation is associated with early repolarization. J Am Coll Cardiol. 2013;62:1015-9.

17. Nam GB, Kim YH, Antzelevitch C. Augmentation of J waves and electrical storms in patients with early repolarization.
N Engl I Med 2008:358:2078-9.

18. KoncZ I, Gurabi Z, Patocskai B, et al. Mechanisms underlying the development of the electrocardiographic and arrhythmic manifestations of early repolarization syndrome. I Mol Cell Cardiol. 2014;68:20-8.

19. Ghosh $\mathrm{S}$, Cooper DH, Vijayakumar R, et al. Early repolarization associated with sudden death: insights from noninvasive electrocardiographic imaging. Heart Rhythm. 2010;7:534-7.

20. Haïssaguerre M, Nademanee $K$, Hocini M, et al. Depolarization versus repolarization abnormality underlying inferolateral J-wave syndromes: new concepts in sudden cardiac death with apparently normal hearts. Heart Rhythm. 2019;16:781-90.

21. Haïssaguerre M, Hocini M, Cheniti G, et al. Localized structural alterations underlying a subset of unexplained sudden cardiac death. Circ Arrhythm Electrophysiol. 2018:11:e006120.

22. Priori SG, Blomström-Lundqvist C, Mazzanti A, et al. 2015 ESC Guidelines for the management of patients with ventricular arrhythmias and the prevention of sudden cardiac death: the Task Force for the Management of Patients with Ventricular Arrhythmias and the Prevention of Sudden Cardiac Death of the European Society of Cardiology (ESC). Endorsed by: Association for European Paediatric and Congenital Cardiology (AEPC). Eur Heart J. 2015;36:2793-867.

23. Belhassen B, Glick A, Viskin S. Excellent long-term reproducibility of the electrophysiologic efficacy of quinidine in patients with idiopathic ventricular fibrillation and Brugada syndrome. Pacing Clin Electrophysiol. 2009;32:294-301.

24. Haïssaguerre M, Sacher F, Nogami A, et al. Characteristics of 
recurrent ventricular fibrillation associated with inferolatera early repolarization role of drug therapy. J Am Coll Cardiol.

$$
\text { 2009;53:612-9. }
$$

25. Antzelevitch C, Yan GX, Ackerman MJ, et al. J-wave syndromes expert consensus conference report: emerging concepts and gaps in knowledge. Europace. 2017:19:665-94.

26. Huelsing DJ, Pollard AE, Spitzer KW. Transient outward current modulates discontinuous conduction in rabbit ventricular cell pairs. Cardiovasc Res. 2001;49:779-89.

27. Huelsing DJ, Spitzer KW, Pollard AE. Spontaneous activity induced in rabbit Purkinje myocytes during activity induced in rabbit Purkinje myocytes during 2003;59:620-7.

28. Tanaka H, Kinoshita O, Uchikawa S, et al. Successful prevention of recurrent ventricular fibrillation by intravenous isoprotereno of recurrent ventricular fibrillation by intravenous isoprotereno
in a patient with Brugada syndrome. Pacing Clin Electrophysiol.
2004;4:1293-4.

29. Bun SS, Maury P, Giustetto C, Deharo JC. Electrical storm in short-QT syndrome successfully treated with isoproterenol. I Cardiovasc Electrophysiol. 2012;23:1028-30.

30. Belhassen B, Glick A, Viskin S. Efficacy of quinidine in high-risk Bethassen B, Glick A, Viskin S. Efficacy of quinidine in high-risk
patients with Brugada syndrome. Circulation. 2004;110:1731-7. 31. Gaita F, Giustetto C, Bianchi F, et al. Short QT syndrome: pharmacological treatment. J Am Coll Cardiol. 2004;43:1494-9. 